\title{
Improving Student Interaction with Chemical Engineering Learning Tools: Screencasts and Simulations
}

\section{Dr. Garret Nicodemus, University of Colorado, Boulder}

Senior researcher at the University of Colorado Boulder in the Department of Chemical and Biological Engineering. Received PhD in Chemical \& Biological Engineering at CU Boulder in 2009 and B.S. in Chemical Engineering at Lafayette College in 2004. Has taught Material \& Energy Balances, Fluid Mechanics, Separations and Mass Transfer, and Senior Process Design.

\section{Prof. John L. Falconer, University of Colorado Boulder}

John L. Falconer is the Mel and Virginia Clark Professor of Chemical and Biological Engineering and a President's Teaching Scholar at the University of Colorado Boulder. He has published more than 225 papers and has 12 patents in the areas of zeolite membranes, heterogeneous catalysis, photocatalysis, and atomic and molecular deposition. He has directed the effort at the University of Colorado to prepare screencasts, ConcepTests, and interactive simulations for chemical engineering courses (www.LearnChemE.com).

\section{Dr. Will Medlin, University of Colorado, Boulder}

J. Will Medlin is an associate professor of Chemical and Biological Engineering and the ConocoPhillips Faculty Fellow at the University of Colorado. He teaches courses in kinetics, thermodynamics, and material and energy balances. His research interests are in the area of surface science and heterogeneous catalysis.

Mrs. Katherine Page McDanel, University of Colorado Boulder, Department of Chemical \& Biological Engineering Dr. Jeffrey Steven Knutsen, Department of Mechanical Engineering, University of Colorado at Boulder

My interests focus primarily on the implementation of novel teaching strategies in the classroom, especially the use of electronic resources such as concept tests, screencasts, and the use of a tablet computer to facilitate a more dynamic presentation of course material. I am currently developing a number of screencasts to eventually facilitate a "flipped classroom" that moves lectures outside the classroom via a series of short videos. Class time is then available for more valuable activities such as discussions of conceptual questions, workshops, and projects. I am especially interested in promoting more open-ended problems, which better approximate engineering challenges that extend beyond the classroom environment. Thus far my favorite courses include Fluid Mechanics, Heat Transfer, Thermodynamics, Dynamics, and Design. 


\title{
Improving Student Interaction with Chemical Engineering Learning Tools: Screencasts and Simulations
}

\begin{abstract}
Interactive screencasts and interactive simulations were prepared and made openly available online to increase student interaction with chemical engineering teaching resources. Screencasts are short (typically less than $10 \mathrm{~min}$ ) videos made using screen capture software and voice narration. Interactive screencasts pose conceptual questions that the viewer can answer by clicking on the answer within the video. Selecting an answer opens another video. If they selected the incorrect answer, the video indicates this and it may give them a hint or tell why the answer is incorrect. Then it allows them to select another answer, which opens another video. This continues until they choose the correct answer; that video then explains why the answer is correct. Similarly, interactive Mathematica simulations allow users to manually change variables and almost instantly see the effects on the system behavior. This encourages student interaction with part of an assignment, and can also be used in class where students are asked to predict system behavior. More than 400 chemical engineering simulations, prepared by a number of authors, are available on the Wolfram Demonstrations Project website. We have prepared about 25 of these simulations and are starting to prepare screencast that explain their use.
\end{abstract}

\section{Screencasts}

A number of studies have shown screencasts to be effective learning materials that are better than lectures. A meta-analysis by the Department of Education [1] found that students who used both online learning and face-to-face instruction performed better than students with only faceto-face instruction. Students with only online learning courses also performed modestly better than those receiving face-to-face instruction. This study analyzed 1,000 studies carried out over twelve years and concluded that a combination of elements resulted in additional learning time, and the additional time produced the increased learning. They stated: "Online learning is more conducive to the expansion of learning time than is face-to-face instruction."

One significant advantage of screencasts is that students really like them. As we have reported for chemical engineering screencasts [2,3], responses in our classes have been overwhelmingly positive. In three courses (chemical engineering material and energy balances, mechanical engineering thermodynamics, and a general engineering computing class), $95 \%$ of students indicated that they felt more confident about the material after watching a screencast. Two comments from end-of-semester student surveys provide an indication of how students like screencasts:

- "They are a useful way to gain more guided practice, in addition to what we learn in class. The major benefit for me is that they are available 24/7, cover a range of topics in all of our classes, and present problems that we haven't seen before."

- "Instead of passively reading an example problem on my own, screencasts are not only another valuable learning resource, but also can actively involve me in problems and concepts in a more engaging medium that incorporates the guidance and insights of an instructor." 
One indication of students' interest in watching screencasts is the fact that they were watched/downloaded more than 1.8 million times in 2013. Garrigus [4] pointed out that students learn best in brief sessions; this is an important incentive for short screencasts. He said that students seek out screencasts on their own, indicating the value to them. Moreover, students liked the control and the portability of screencasts. He observed that a 10-minute screencast covered the material that he would present in 20-minute class, so screencasts can be more efficient. Toto [5] studied the use of screencasts to supplement a first-semester, general chemistry class for distance learners in 2006 . He created 60 screencasts that specifically addressed concepts from homework assignments that students scored poorly on the year before. When Toto compared student performance between the classes, he found that students with access to the screencasts scored $11.2 \%$ better in the course overall and $21.8 \%$ better on the difficult concepts that prior students scored poorly on.

\section{Interactive screencasts}

One suggestion from end-of-semester surveys is to include online quizzes with the screencasts so that students could test their understanding of the material. To improve student interaction with screencasts, we developed about 80 interactive screencasts for two courses. These screencasts pose a conceptual multiple-choice problem and then allow students to select an answer, which then opens another short screencast that tells if the answer is correct. If correct, the screencasts explains why. If incorrect, it may provide a hint or suggestion what to think about before selecting another answer within that screencasts. A snapshot of an interactive screencast is shown in Figure 1.

These interactive screencasts can be used as:

- pre-class assignments that promote critical thinking. The screencasts can be linked with reading assignments or other screencasts to provide students a method to quiz their understanding prior to applying it in class.

- review materials for exams. Viewing of this type of screencasts doubled during exam weeks for our thermodynamics course.

- components of a learning module. Interactive screencasts can be combined with other resources as part of online courses.

- resources for identifying student issues with material and misconceptions. Data collected from student choices can help focus teaching efforts. 


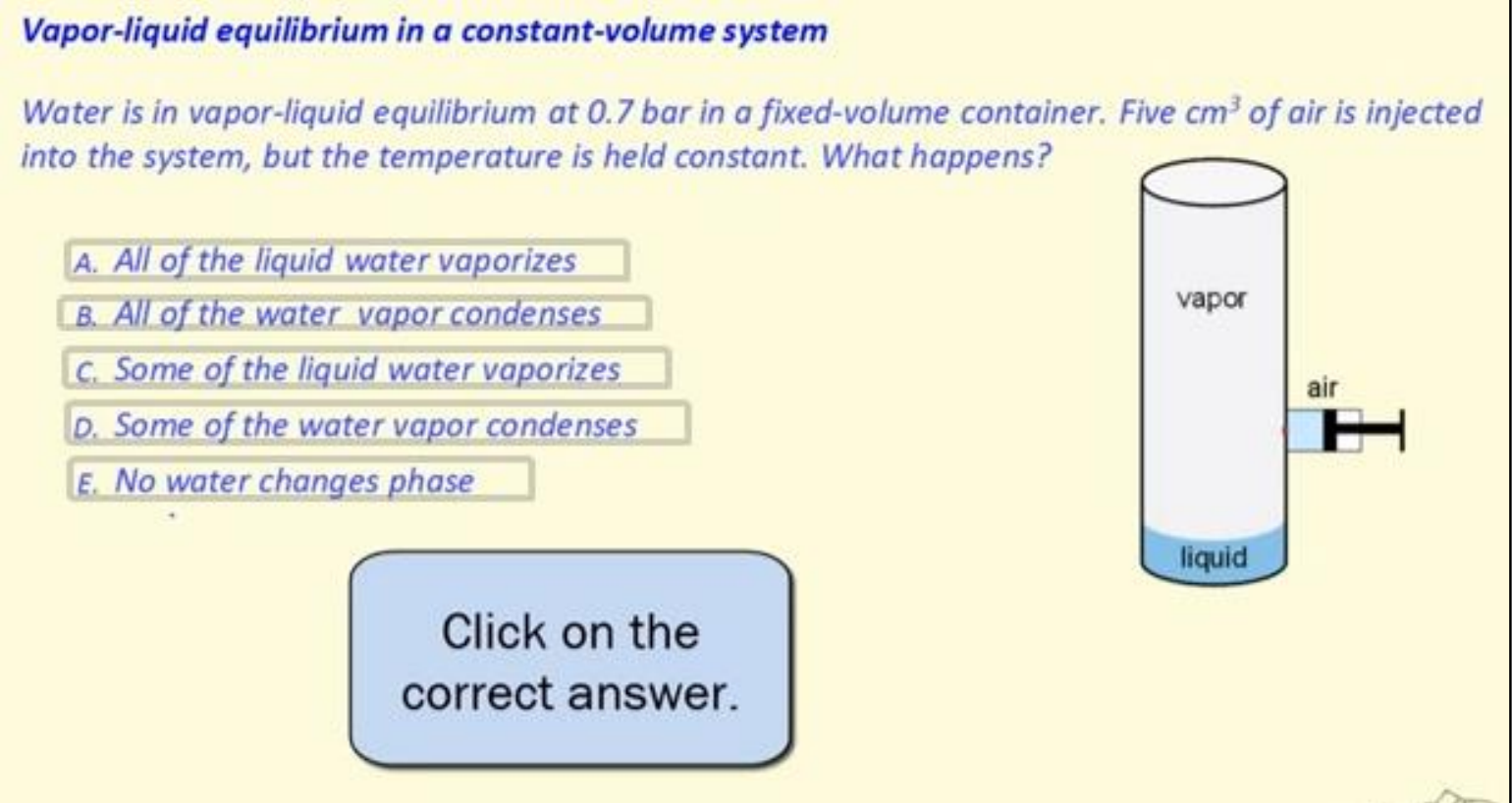

Figure 1. A thermodynamics interactive screencast (http://youtu.be/9_ZuvV-Cl4E). The answers indicated are hyperlinks, each of which opens a screencast corresponding to that answer.

Interactive screencasts are prepared in one recording using Camtasia Studio. The question is presented and a response to each answer is recorded. Incorrect answers often contain pointers on how to approach the problem or things to consider in analyzing the system. Correct answers explain why the answer is correct. This single recording is then edited and produced as separate mp4 video files that are uploaded to YouTube, which allows videos to be linked to other videos.

Over 40 videos in fluid mechanics and 40 in thermodynamics were prepared and placed on YouTube (/learncheme). They are also organized by topics on our website (www.learncheme.com). These interactive screencasts were watched over 24,000 times in fall 2013, and they received positive feedback from students surveyed at the end of a summer fluid mechanics course. Some comments from students were:

- "I liked the interactive screencasts the most because I could get immediate feedback as to how well I knew the material -- I also REALLY liked these since if you picked the wrong answer, it would explain WHY your answer wasn't right."

- "Amazing videos, keep up the good work!"

- "I like the interactivity!"

- "Please make more of these. I wish I would have had them in xx class."

Once the screencasts have been used enough times, the answers selected for each question may allow us to better identify misconceptions about the topic.

\section{Interactive simulations}

An effective approach to demonstrate the behavior of a chemical engineering system is to use an interactive simulation that allows the user to manipulate variables and observe the effects on the system behavior. They can be designed to focus on a specific concept or model a complex unit process. Student interaction with computer simulations is known to have positive effects on learning [6-8] and promote student inquiry and exploration [9, 10]. Virtual labs created for 
chemical engineering also promote this type of learning without the large expenses necessary for the "real-world" labs [11-13].

Some desirable traits for simulations are ease of programming and ease of using the simulation. Wolfram developed the open-code Demonstrations Project (http://demonstrations.wolfram.com) where interactive simulations can be posted after review by Wolfram. One advantage of these simulations is that they can be prepared with minimal effort because of the built-in functions in Mathematica [14]. Wolfram provides screencasts and workbook tutorials to learn the skills necessary to prepare interactive simulations [15]. The Wolfram Demonstrations Project has more than 9,000 interactive simulations, and more than 400 chemical engineering simulations that can be used on computers without a Mathematica license.

Many of the Mathematica simulations for chemical engineering are solutions to differential equations (ordinary and partial) or algebraic equations. The output is plotted graphically and/or presented through visual animation, and many simulations allow switching between outputs. One of the key features of these simulations is that the calculations are done in real-time as input parameters are manipulated using user-friendly controls. An example simulation is shown in Figure 2. The controls in this simulation are sliders shown at the left side of the figure; these sliders can be moved to change the indicated variables, and the new graph is plotted almost instantaneously. Other types of controls include buttons, check boxes, location drag, tabs, figure zoom, and animation. Simulation code and publishing information are submitted to the Wolfram Demonstrations Project for review and feedback prior to publishing online. The final online versions allows users to either run them online using a browser plugin or to download the executable version to use offline (without Mathematica).

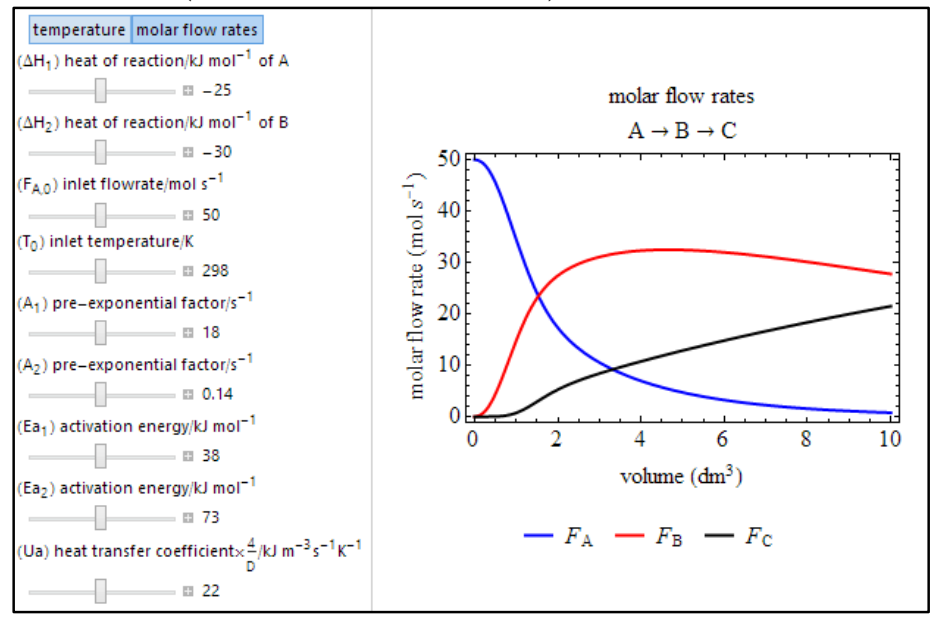

Figure 2. Simulation demonstrating relationships between variables and system behavior. http://demonstrations.wolfram.com/SensitivityOfAPlugFlowReactorToModelParameters/

\section{Using the simulations in class}

One way to use these simulations is to ask students to predict the behavior of a system. For example, this could be done with student-held clickers. An example from kinetics is shown in Figure 3. In this question, a plug flow reactor with heat transfer through the walls is used for an exothermic gas-phase reaction. The temperature versus distance down the reactor is plotted. The ConcepTest asks which plot represents the new temperature versus distance plot if the total molar flow rate into the reactor is doubled, but other parameters do not change. Four possible outputs are shown; they were generated with the simulation by changing variables. 

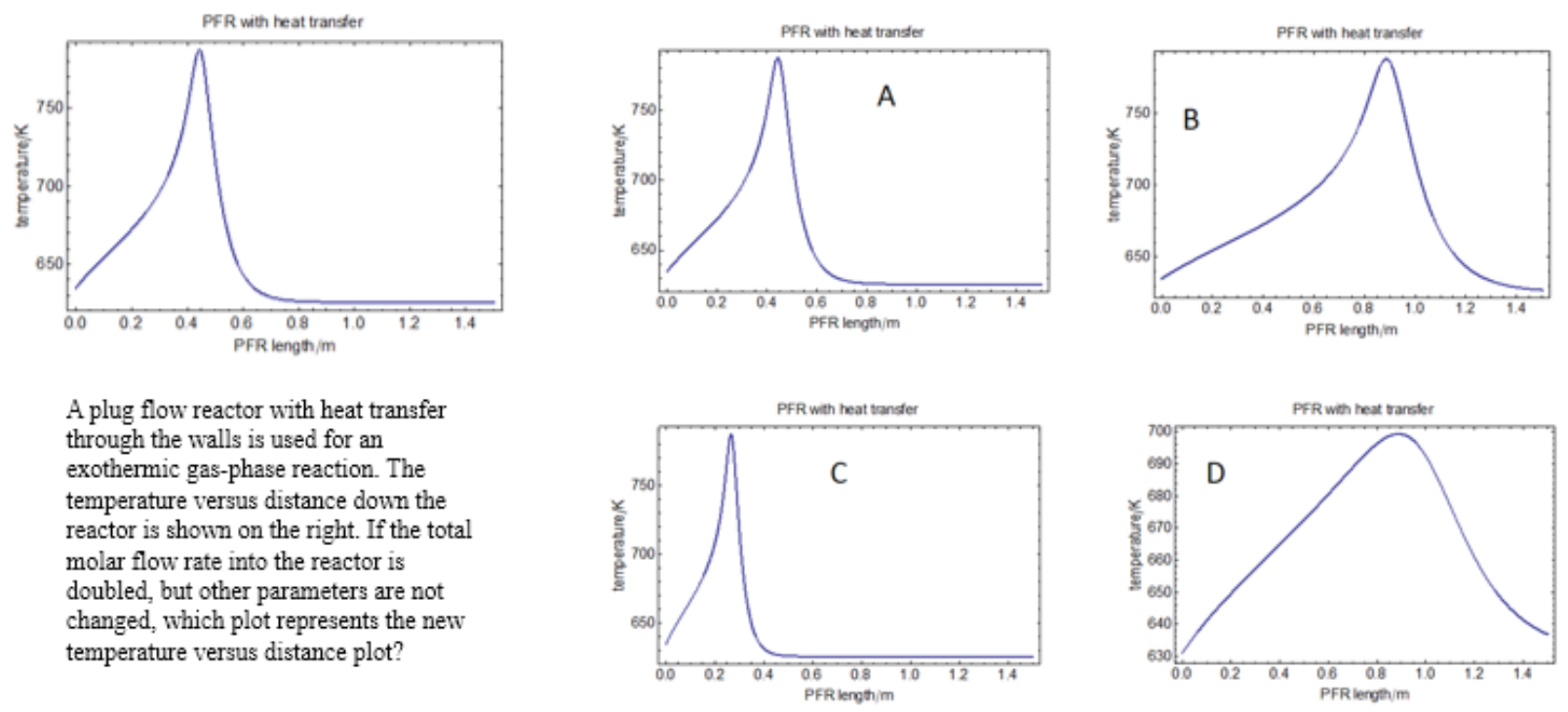

Figure 3. ConcepTest designed using a simulation for a plug flow reactor with heat exchange. http://demonstrations.wolfram.com/ParametricSensitivityOfPlugFlowReactorWithHeatExchange/

After the students select their answers and then discuss the answers with their classmates, the simulation is used in class to demonstrate the behavior. In this particular case, doubling the inlet flow rate means that the same curve shape is seen, but spread over twice the distance. The real advantage of the simulation is that the system behavior can be visually presented and can describe more than one process condition. Follow up questions can be given to assess the effects of other variables or apply the same concept to other systems. Other ways the simulations can be used in class include:

- Discussing parametric sensitivity of a system. Instead of demonstrating one example with one set of conditions, a simulation can cover ranges of variables with simple slider controls.

- Showing system behavior and having students elucidate mathematical relationships. Experimental conditions can be mimicked using a simulation and students can work together to determine relationships between variables.

- Preparing for class work. Pre-class exposure to system behavior can promote students critical thinking prior to class.

- Discussing system behavior in homework assignments. Students can use simulations to help describe physical phenomena or they can design simulations to model systems presented in homework problems.

More than 400 interactive simulations are currently available for chemical engineering topics (http://demonstrations.wolfram.com/search.html?query=chemical\%20engineering). Many of these simulations are specific examples that may be best suited for advanced topics. However, a significant number focus on core concepts, and the open source nature of the simulations allows for easy manipulations to the code to modify them if desired. The Wolfram site currently has more than 9,200 interactive simulations that cover a range of fields including engineering, science, mathematics, and computation. They are searchable and subdivided by general topics such as mechanical, civil, and chemical engineering. We have created links to the simulations on www.LearnChemE.com where we have further subdivided the chemical engineering simulations by course topics to make them easier to use. 
Interactive simulations from the Wolfram site were used in a 5-week summer session of fluid mechanics. They were used in pre-class quizzes to introduce concepts to students with questions like, "What happens when..." and "Where is the point in which...". Simulations were also used in class to demonstrate a system and support an example problem performed in class. They were also used in conjunction with a ConcepTest to ask students to predict system behavior when conditions were changed. An advantage of using them in class is to help students see relationships before doing calculations. Some of the common comments from students with regards to the simulations were:

"The demos were a great way to visualize a concept and analyze the relationship between an equation or theory and the variables involved."

"The Wolfram demos were helpful because I could see how things changed instead of just having one question and one answer like in a quiz, clicker, or screencast."

"The demo could use better explanations of what is being observed and controlled. It was confusing at first to determine this on our own."

A consistent concern expressed by a number of students is that many of the simulations are not explained sufficiently to make them easy to use: the system is not described sufficiently, and often the plots and sliders contain variables that are depicted as symbols and not described clearly. Indeed many of the simulations on the Wolfram site have brief descriptions, and this was part of our motivation to prepare our own simulations. We have provided more detailed descriptions of our simulations, and in some cases prepared screencasts that explain the system and how to use the simulation. Thus, as more of our simulations are completed, we expect students will find them easier to use.

\section{Conclusions}

To increase student interaction with materials outside of class, both interactive screencasts and interactive simulations were designed. Interactive screencasts provide students the ability to quiz themselves and hear experts explain both correct and incorrect responses to these conceptual questions. Interactive simulations designed using Mathematica provide visual representations of system behavior. They can be used without purchasing software and a repository of over 400 simulations related to chemical engineering topics are available at the Wolfram Demonstrations site. These simulations can be used both in and out of class to promote student interaction with the material.

\section{Acknowledgments}

We gratefully acknowledge support from NSF Grant DUE 1244183 and thank Rachael L. Baumann for preparing many of the interactive simulations that we posted on the Wolfram Demonstration site.

\section{References}


1. B. Means, Y. Toyama, R. Murphy, M. Bakia, K. Jones, Evaluation of Evidence-Based Practices in Online Learning: A Meta-Analysis and Review of Online Learning Studies, U.S. Department of Education (2010). http://www2.ed.gov/rschstat/eval/tech/evidence-based-practices/finalreport.pdf

2. J.L. Falconer, J. deGrazia, J.W. Medlin, M. Holmberg, Using Screencasts in Chemical Engineering Courses, Chem. Eng. Ed. 43, 302-305 (2009).

3. J.L. Falconer, G. Nicodemus, J. deGrazia, J.W. Medlin, Chemical Engineering Screencasts, Chem. Eng. Ed., in press (2012).

4. J. Garrigus, How can screencasts improve active learning? http://blog.uta.edu/bpn/2010/02/23/how-canscreencasts-improve-active-learning/

5. J. Toto, K. Booth, Effects and implications of mini-lectures on learning in first-semester general chemistry, Chem. Ed. Resch. Pract. 9, 259-266 (2008).

6. D. Bodemer, R. Ploetzner, I. Feuerlein, and H. Spada. "The active integration of information during learning with dynamic and interactive visualization”, Learn Instr. 14, 325, 2004.

7. J. van der Meij and T de Jong. "Supporting students learning with multiple representation in a dynamic simulation-based environment", Learn Instr. 16, 199, 2006.

8. Kadiyala, M., and B. Crynes, "A review of literature on effectiveness of use of information technology in education”, J. Eng. Ed., 89 (2) 177, 2000.

9. M. R. Lepper and T.W. Malone. "Intrinsic motivation and instructional effectiveness in a computer-based education", Aptitude, Learning, and Instruction, edited by R.E. Snow and M.J. Farr (Lawrence Erlbaum Associates), Hillsdale, NJ, 1987.

10. N. S. Podolefsky, K. K. Perkins, and W. K. Adams. "Factors promoting engaged exploration with computer simulations", Phys. Rev. ST Phys. Educ., Res. 6, 020117, 2010.

11. M.G. Rasteiro et al. "LABVIRTUAL-A virtual platform to teach chemical processes", Education for Chemical Engineers, Volume 4, Issue 1, April 2009.

12. S. Vaidyanath, J. Williams, M. Hilliard, T. Wiesner. "The development and deployment of a virtual unit ops laboratory”, Chem. Eng. Ed, 41 (2), 144-152, 2007.

13. M.D. Koretsky, C. Kelly, and E.S. Gummer. "Student Learning in Industrially Situated Virtual Laboratories", Chem. Eng. Ed., 45(3), 219-228, 2011.

14. P. Mokhasi, J. Adduci, and D. Kapadia. "Understanding differential equations using Mathematica and interactive demonstrations", CODEE Journal. http://www.codee.org/ref/CJ12-3093 (accessed December 23, 2013)

15. "New in 7.0: Dynamic Interactivity." —Wolfram Mathematica 9 Documentation. Wolfram, n.d. Web. 22 Oct. 2013. 